\title{
Gait analysis in demented subjects: Interests and perspectives
}

\author{
Olivier Beauchet' \\ Gilles Allali² \\ Gilles Berrut ${ }^{3}$ \\ Caroline Hommet ${ }^{4}$ \\ Véronique Dubost ${ }^{5}$ \\ Frédéric Assal ${ }^{2}$ \\ 'Department of Geriatrics, Angers \\ University Hospital, France; \\ ${ }^{2}$ Department of Neurology, \\ Geneva University Hospital, \\ France; ${ }^{3}$ Department of Geriatrics, \\ Nantes University Hospital, France; \\ ${ }^{4}$ Department of Internal Medicine and \\ Geriatrics, Tours University Hospital, \\ France; ${ }^{5}$ Department of Geriatrics, \\ Dijon University Hospital, France
}

\begin{abstract}
Gait disorders are more prevalent in dementia than in normal aging and are related to the severity of cognitive decline. Dementia-related gait changes (DRGC) mainly include decrease in walking speed provoked by a decrease in stride length and an increase in support phase. More recently, dual-task related changes in gait were found in Alzheimer's disease (AD) and non-Alzheimer dementia, even at an early stage. An increase in stride-to-stride variability while usual walking and dual-tasking has been shown to be more specific and sensitive than any change in mean value in subjects with dementia. Those data show that DRGC are not only associated to motor disorders but also to problem with central processing of information and highlight that dysfunction of temporal and frontal lobe may in part explain gait impairment among demented subjects. Gait assessment, and more particularly dual-task analysis, is therefore crucial in early diagnosis of dementia and/or related syndromes in the elderly. Moreover, dual-task disturbances could be a specific marker of falling at a pre-dementia stage.
\end{abstract}

Keywords: gait, prediction of dementia, risk of falling, older adult

The main clinical hallmark of dementia is cognitive decline (Waldemar et al 2007). Although not as prominent, motor disorders are commonly described in later stages of dementia (Camicioli et al 1998; Marquis et al 2002; Sheridan et al 2003; Scarmeas et al 2005; Waite et al 2005) and include gait apraxia, bradykinesia, extrapyramidal rigidity, resting tremor and various gait disorders such as cautious gait or gait slowing (Camicioli et al 1998; Goldman et al 1999; Marquis et al 2002; Sheridan et al 2003; Scarmeas et al 2005; Waite et al 2005; Waldemar et al 2007). Recently, a few studies have shown that motor disorders, and specifically gait disorders, may be present at an early stage of dementia (Camicioli et al 1998; Verghese et al 2002, 2007a; Scarmeas et al 2005; Waite et al 2005). There is an increased interest in the study of these dementia-related gait changes (DRGC) since they could be used to improve early diagnosis and better understand risk of falling in subjects with dementia or even at a pre-dementia stage (Morgan et al 2007).

\section{Methods}

The literature was searched using Medline database. General inclusion criteria included meta-analysis, randomized controlled trial, controlled clinical trial, guideline and review articles published in English in peer-reviewed priority journals. Keywords used in PubMed were the following: Aged; AND aged, 80 over; AND Gait disorders; AND Dementia. For completeness, additional key trials known to the authors that did not meet the initial search criteria were included. 
A total of 40 papers met the criteria (116 were identified by PubMed, but 76 did not correspond to the topic of the paper), 23 additional papers already known by the authors were also reviewed.

\section{Gait changes in demented subjects: Evidence for a relationship}

Dementia and gait disorders represent a major public health issue because of their high prevalence, their related adverse outcomes leading to loss of autonomy and independence, and their high costs for public health and social services (Alexander 1996; Allan et al 2005; Burn et al 2006). It has been shown that demented older adults present with greater gait impairments than those expected as a result of the normal aging process (Alexander 1996; Allan et al 2005; Burn et al 2006; Scherder et al 2007). Different DRGC have been described, mostly when comparing subjects with Alzheimer's disease (AD) versus healthy control subjects. The walking speed decreases in AD and further parallels severity of the disease (Van Iersel et al 2004, 2007; Morgan et al 2007; Scherder et al 2007). This change in velocity has been related to a decrease in stride length and an increase in support time (Van Iersel et al 2004). Furthermore, subjects with vascular dementia (VaD) and dementia with Lewy bodies (DLB) walked more slowly and presented a reduced step length than AD subjects (Allan et al 2005; Merory at al 2007). DRGC are not specific to any type of dementia as similar changes in gait pattern have been reported with advanced age (Alexander 1996; Scherder et al 2007).

The origins of DRGC are not only associated to classic motor disorders in the basal ganglia, cerebellum and primary motor areas, but also to central misprocessing of information that is required to maintain gait safety and that include attention and executive functions (Goldman et al 1999; Sheridan et al 2003; Beauchet et al 2006). Most studies exploring DRGC have focused on mean values of stride parameters (Alexander 1996; Van Iersel et al 2004). Recently, an increase in stride-to-stride variability while usual walking and dual-tasking has been shown to be more specific than any change in mean value in patients with dementia (Sheridan et al 2003; Hausdorff 2004; Beauchet et al 2005a; Webster et al 2006; Allali et al 2007; Verghese et al 2007b). This stride-to-stride variability is a fine marker of gait control and, thus, highlights that gait should not be considered as a simple automatic motor behavior, but as a rather complex and higher level of cognitive functioning (Hausdorff 2004; Beauchet et al 2005a; Allali et al 2007). Exploring strideto-stride variability in demented subjects represents a new way to access gait disorders related to higher levels of gait control impairment.

\section{Early diagnosis of dementia: Gait analysis as a new method of expertise?}

Whatever its etiology, treatments of dementia do not cure the disease, although its evolution may be slowed down (Garcia et al 2007). Available treatments such as acetylcholinesterase inhibitors (AchEIns) or memantine are efficient on cognitive symptoms and on global evolution for a limited period of time (Birks 2006; Garcia et al 2007), and their efficiency depends on early administration (Birks 2006).

Alzheimer's disease is the main cause of dementia (Ferri et al 2005; Rimmer et al 2005; Small et al 2007). Although operational criteria do exist, it has been shown that within subjects with AD-related cognitive decline only 50\% are diagnosed with the disease, and only $30 \%$ at an early stage (Ferri et al 2005; Rimmer et al 2005). This may principally be explained by unclear boundaries between early dementia syndrome and normal aging. Neuropsychological deficits including memory, attention and executive functions are frequently affected first (Ferri et al 2005; Rimmer et al 2005; Small et al 2007), but heavily rely on psychometrics, which are time consuming and lack specificity in terms of predicting the occurrence of dementia syndrome. If defining pathological cognitive aging seems quite straightforward in the presence of dementia according to DMS-IV criteria, it becomes much more complex for intermediate cognitive impairment such as age-related cognitive decline (Ferri et al 2005) or mild cognitive impairment (MCI) as risk factors for AD (Rimmer et al 2005; Di Carlo et al 2007; Visser and Verhey 2007) but these are not specific to cognitive aging and hence cannot be used for early diagnosis (Rimmer et al 2005).

The question is then to determine whether the specificity of cognitive expertise in the early diagnosis of AD may be improved. Over the past ten years, a few studies demonstrated that cortical gait control impairment and certain motor deficits were one of the early signs of AD and non-Alzheimer dementia (Camicioli et al 1998; Scarmeas et al 2005; Waite et al 2005; Verghese et al 2007a). Rhythmic motor tasks such as "finger tapping" and walking were performed more slowly in subjects developing cognitive decline than in those with no cognitive impairment whereas a one-second increase compared with the mean score of healthy subjects was associated with a risk of cognitive decline increased by a factor of 1.26 (OR = 1.26; IC = 1.01-1.60) (Camicioli et al 1998). 
In the same study, a motor coordination impairment, ie, the capacity to alternately repeat movements of the lower limbs, was associated with a high risk of developing cognitive decline $(\mathrm{OR}=6.10 ; \mathrm{IC}=1.40-26.30)$. In a longitudinal cohort of 630 community-dwelling participants aged 75 or over at recruitment, subjects with cognitive impairment associated with gait and motor slowing were the most likely to develop dementia (OR 5.6; 95\% CI 2.5-12.6) (Waite et al 2005). Recently, Verghese and colleagues (2007a) presented similar findings in 427 subjects aged 70 and older, and concluded that quantitative gait measures might predict future risk of cognitive decline and dementia in initially non-demented older adults. These recent data suggest that an early diagnosis of dementia and/or related syndromes in the elderly should include DRGC analysis.

\section{Gait disorders related to cortical gait control impairment: Interest in gait analysis under dual-task conditions}

Walking is classically described as an automatic, rhythmic, and regular motor activity characterized by alternated, coordinated movements of crossed flexion-extension of the lower limbs while steady-state walking (Nutt et al 1993). It is considered as a simple motor activity in healthy subjects because of its predominant automatic character acquired during the simultaneous maturation of the locomotor and nervous systems. From a neurological viewpoint, the acquisition of such automatisms relies on motor procedural memory, allowing for the gradual appearance of motor processes enabling the automation of walking in healthy adults. Motor procedural memory handles information based on action rules (Beaunieux et al 2006; Squire et al 1990). Its expression is implicit, and cannot be dissociated from action.

In experimental psychology, the adaptative control of thought (ACT) by Anderson and Fincham (1994) is considered as a reference. It distinguishes three successive phases during which the subject generates, combines, and improves a specific procedure for a given task. The first phase is the declarative phase, corresponding to the selection of relevant information for the requested task, and relies on intellectual abilities, as well as on important attentional resources. In the case of a motor action, it may be assimilated to the motor plan elaboration step. The second phase, called the knowledge compilation phase, corresponds to the transformation of declarative information into procedures, and may be assimilated to the elaboration of the motor program. Finally, the third and final phase is the procedural phase, corresponding to the adjustment and automation of the procedure.

The procedural learning of walking enables the automatic, unconscious triggering of underlying motor programs in healthy adults. Such an implicit character is predominant in walking and implies that walking only requires limited attentional resources in healthy subjects (Beauchet et al 2005a; Dubost et al 2006, 2007). Although this assumption has been confirmed in young subjects (Beauchet et al 2005b), several studies proved it was not the case in older adults and demented subjects, in which cortical gait control is involved while walking (Dubost et al 2006, 2007). Furthermore, the most ordinary walking conditions demonstrate that the implicit character of walking is not univocal: stride-to-stride time modifications, the presence of obstacles or directional changes constitute intentional parameters requiring explicit cognitive function.

The clinical test used to highlight cortical involvement in gait control is based on a dual-task paradigm, the principle of which is that the subject performs an attention-demanding task while walking and to observe any walking modification compared with the reference task, ie, usual walking (Abernethy 1988; Woollacott and Shumway-Cook 2002). Dual-task paradigms are based on the hypothesis that two simultaneously-performed tasks interfere if relying on identical functional and/or cerebral subsystems (Woollacott and Shumway-Cook 2002). In the case of a paradigm including walking and an attention-demanding task, the interference is based on the hypothesis of a joint involvement of attention (Abernethy 1988). The primary task therefore is the "attention-demanding" task, while the secondary task is represented by walking. The interferences observed are modifications of the performance in one or both tasks, which are measured by comparing the performances under single- and dual-task conditions (Abernethy 1988; Pashler 1994; Woollacott and Shumway-Cook 2002). Gait modifications are then interpreted as the involvement of attention while walking, and therefore attest of a level of cortical control ensuring the functionality of walking, while making control frailer.

Attention is a complex and multidimensional cognitive function that overlaps with executive functions and one dimension of which participate in improving the processing of information (Treisman 1969). Attention is a limited resource in cognitive processing that may be overloaded by two competing tasks when performed simultaneously, or dual-tasking (Abernethy 1988; Woollacott and ShumwayCook 2002), then consecutively leading to a decline of performance in one or both tasks. Two categories of interferences 
have been defined (Abernethy 1988; Pashler 1994). The first one is called capacity interference and is the result of a central overload due to involvement of different information processes requiring attention. In contrast with capacity interference, structural interference is defined as a peripheral overload due to an inability to perform simultaneously two tasks involving the same category of information. The bottleneck and cross-talk models are based on this theoretical approach. Although similar tasks performed simultaneously provoke a decline in performance in the bottleneck model, the cross-talk model assumes that task similarity reduces interferences, thus leading to better performance.

Dual-task related gait changes reflect the capacity to appropriately allocate attention between two tasks performed simultaneously and, therefore, are related to executive function efficiency (Pashler 1994; Sheridan et al 2003; Allali et al 2007). Alzheimer's subjects with moderate dementia and executive dysfunction presented with high gait variability suggesting that this gait parameter could be a sensitive and specific marker of frontal cortical control of walking (Sheridan et al 2003; Allali et al 2007). In another sample, the degree of efficiency of the executive function was correlated to the degree of stride time variability (Hausdorff et al 2005). Recently, the disruption of stride-to-stride variability was shown to be the most significant gait parameter while dual-tasking in a sample of older subjects with a dysexecutive syndrome (Allali et al 2007).

\section{Dementia and the risk of falling: Gait analysis as the key test}

Dementia, even at an early stage and without any other neurological signs, is a risk factor of falling that is constantly observed, irrespective of the subject's living environment (Shaw 2002). The incidence of falls is significant in demented subjects and is approximately two or three times higher than in subjects without cognitive decline (Alexander 1996; Shaw 2002). Additionally, cognitive decline, including dementia or not, has been associated with static balance or gait disorders directly related to the severity of cognitive decline (Alexander 1996; Allan et al 2005; Burn et al 2006). When comparing subjects with mild to moderate $\mathrm{AD}$ to healthy subjects that were matched for age and sensory deficits, the performance of unipodal support or the tandem stance was associated to the severity of cognitive deficits (Frassen et al 1999). Additionally, when testing the coordination of lower limbs in the sitting position while carrying out a test of the « Tapping » type, the performance observed during this test also decreased according to the degree of cognitive impairment.
Depending on how severe cognitive decline is, associated neurological anomalies such as extrapyramidal syndrome have been observed, which explains the exponential increase in disorders and the incidence of falls (Shaw 2002).

The most frequently identified explanatory factor in demented subjects is postural instability, irrespective of the nature of dementia (Visser 1983; Nakamura 1996; O’Keeffe 1996; Buchner et al 1997; Frassen et al 1999). Subjects with VaD or DLB obviously are at an increased risk of falling compared to subjects with AD (Visser 1983; Sattin 1992; Clemson et al 1996; Nakamura 1996; O'Keeffe 1996; Buchner et al 1997). The role of environment in the mechanism of falls in older demented subjects remains controversial, just as in older subjects without dementia. Few studies have reported an association between falls and the environment, while others were unable to demonstrate such relation (Sattin 1992; Clemson et al 1996; Carter et al 1997; Close et al 1999).

It has been shown that gait characteristics while usual walking could be related to an executive function deficit. Reduced walking speed was related to poor performances in the Trail Making Test A and B, a neuropsychological test evaluating executive function, in a group of 926 communityliving older subjects, under obstacle avoidance conditions, that is considered as an attention-demanding task (Ble et al 2005). Previous studies were also able to establish such type of relation between executive dysfunction and poor performances in physical tests (Sheridan et al 2003; Hausdorff et al 2005; Allali et al 2007), and explain how interesting it may be to use dual-task paradigms during gait analysis in subjects with dementia. Additionally, in a population of 325 community-living older subjects, a poor performance in the Trail Making Test part B (ie, with practice time over 180 seconds) was associated with a risk of severe trauma in the event of a fall that was 1.9 superior compared with subjects performing the Trail Making Test part B in less than 180 seconds (Nevitt et al 1991).

DRGC usually lead to falls and associated risk factors were recently reviewed from the most to the least important one (Oliver et al 2004): clinically abnormal walking, a history of falling and cognitive decline. Fall-related gait disorders associated with dementia may be prevented since several studies showed that prevention programs had resulted in a 20 to $25 \%$ reduction in falling rates (Tinetti et al 1998; Oliver et al 2003; Tinetti 2003). In addition, criteria have been established in order to better identify subjects at risk of falling (Oliver et al 2003). Consequently, an early identification of potential fallers through gait analysis is an indispensable 
first step of any efficient attempt to prevent falls in subjects with dementia.

\section{The neuronal basis for dementia- related changes in gait}

The automatic, implicit character is predominant in walking. It essentially relies on sub-cortical and spinal control, with a neuronal network localized in the lumbar area (Nutt et al 1993; Dietz 2003). These spinal neuronal networks that include motor neurons and inter neurons are called "central pattern generator" (CPG) for locomotion (Forssberg and Grillner 1973; Forssberg 1979; Dietz 2003). They allow the generation of automatic and rhythmic motor activity patterns, corresponding to alternate, coordinated movements of crossed flexion-extension of the upper limbs, even in the absence of sensory afferences or control by supraspinal structures (Dietz 2003). In humans, several clinical observations corroborate the existence of CPG, such as spontaneous locomotor activity in infants, or persistent rhythmic, coordinated movements of the lower limbs in paraplegic subjects after a medullar section in the dorsal region (Forssberg and Grillner 1973; Forssberg 1979). At the supramedullary level, a particularly important locomotor zone is the pediculopontine nucleus (PPN), localized at the mesencephalic level that is considered to be a site of termination for basal ganglia output, and hence probably plays a key role in the modulation of walking (Pahapill and Lozano 2000).

At the cortical level, there is increased evidence that dysfunction of temporal and frontal lobe may explain motor impairment among demented subjects. It has been shown that temporal atrophy, and specifically the hippocampus, is not only related to memory but also to motor dysfunction (Scherder et al 2007). Furthermore, both clinical studies based on dual-tasking and brain imaging studies have shown that frontal lobe dysfunction may be related to gait disorders in demented subjects. Nakamura and colleagues (1997) demonstrated that increased oscillations of the body in a static position, a reduced stride length and increased stride variability in subjects with $\mathrm{AD}$ could be related to a decreased cerebral perfusion in the frontal lobe.

\section{Recommendations for future research}

Visual observation is usually used for gait analysis by the clinicians but do not give sufficient measures of gait. Objective and accurate gait analysis of temporal and spatial characteristics by validated devices may provide useful information to improve early diagnosis and better understand risk of falling among subjects with cognitive decline. In particular, increase of stride time variability (ie, stride-to-stride variation of gait cycle duration) while dual-tasking has been specifically associated with impaired executive function (IEF) in demented older adults. Acquiring more information about dual-task related changes in stride time variability among demented subjects with and without IEF could add to our understanding of IEF-related gait instability.

\section{Conclusion}

Gait disorders and dementia are frequently associated. There is increase evidence that DRGC are not only attributable to motor disorders, but also associated to problems with the cortical processing of information what may be present at the early stage of dementia. Dysfunction of temporal and frontal lobe may in part explain gait disorders among demented subjects. The main clinical implications are that cortical related changes in gait characteristics could be used to improve the early diagnosis of dementia and detection of an increased risk of falling in demented subjects.

\section{References}

Abernethy B. 1988. Dual-task methodology and motor skills reseasch: somme applications and methodologies contraints. J Hum Mov Studies, 14:101-32.

Alexander NB. Gait disorders in older adults. 1996. J Am Geriatr Soc, 44:434-51.

Allali G, Kressig RW, Assal F, et al. 2007. Changes in gait while backward counting in demented older adults with frontal lobe dysfunction. Gait Posture, 26:572-6.

Allan LM, Ballard CG, Burn DJ et al. 2005. Prevalence and severity of gait disorders in Alzheimer's and non-Alzheimer's dementias. $J$ Am Geriatr Soc, 53:1681-7.

Anderson JR, Fincham JM. 1994. Acquisition of procedural skills from examples. J Exp Psychol Learn Mem Cogn, 20:1322-40.

Beauchet O, Berrut G. 2006. Gait and dual-task: definition, interest, and perspectives in the elderly. Psychol Neuropsychiatr Vieil, 4:215-25.

Beauchet O, Dubost V, Aminian K, et al. 2005a. Dual-task-related gait changes in the elderly: does the type of cognitive task matter? $J$ Mot Behav, 37:259-64.

Beauchet O, Dubost V, Herrmann FR et al. 2005b. Stride-to-stride variability while backward counting among healthy young adults. J Neuroengineering Rehabil, 2:26.

Beaunieux H, Hubert V, Witkowski T, et al. 2006. Which processes are involved in cognitive procedural learning? Memory, 14:521-39.

Ble A, Volpato S, Zuliani G, et al. 2005. Executive function correlates with walking speed in older persons: the InCHIANTI study. $J$ Am Geriatr Soc, 53:410-15.

Birks J. 2006. Cholinesterase inhibitors for Alzheimer's disease. Cochrane Database Syst Rev, 1:CD005593.

Buchner DM, Cress ME, De Lateur BJ, et al. 1997. The effect of strength and endurance training on gait, balance, fall risk, and health services use in community-living older adults. J Gerontol A Biol Sci Med Sci, 52:M218-24.

Burn DJ, Rowan EN, Allan LM, et al. 2006. Motor subtype and cognitive decline in Parkinson's disease, Parkinson's disease with dementia, and dementia with Lewy bodies. J Neurol Neurosurg Psychiatry, 77:585-9. 
Camicioli R, Howieson D, Oken B, et al. 1998. Motor slowing precedes cognitive impairment in the oldest old. Neurology, 50:1496-8.

Carter SE, Campbell EM, Sanson-Fisher RW, et al. 1997. Environmental hazards in the homes of older people. Age Ageing, 26:195-202.

Clemson L, Cumming RG, Roland M. 1996. Case-control study of hazards in the home and risk of falls and hip fractures. Age Ageing, 25:97-101.

Close J, Ellis M, Hooper R, et al. 1999. Prevention of falls in the elderly trial (PROFET): a randomised controlled trial. Lancet, 353:93-7.

Di Carlo A, Lamassa M, Baldereschi M, et al. 2007. CIND and MCI in the Italian elderly: frequency, vascular risk factors, progression to dementia. Neurology, 68:1909-16.

Dietz V. 2003. Spinal cord pattern generators for locomotion. Clin Neurophysiol, 114:1379-89.

Dubost V, Annweiler C, Aminian K, et al. 2007. Stride-to-stride variability while enumerating animal names among healthy young adults: Result of stride velocity or effect of attention-demanding task? Gait Posture, in press.

Dubost V, Kressig RW, Gonthier R, et al. 2006. Relationships between dual-task related changes in stride velocity and stride time variability in healthy older adults. Hum Mov Sci, 25:372-82.

Ferri CP, Prince M, Brayne C, et al. 2005. Alzheimer's Disease International. Global prevalence of dementia: a Delphi consensus study. Lancet, 366:2112-17.

Forssberg H. 1979. On integrative motor functions in the cat's spinal cord. Acta Physiol Scand Suppl, 474:1-56.

Forssberg H, Grillner S. 1973. The locomotion of the acute spinal cat injected with clonidine i.v. Brain Res, 50:184-6.

Franssen EH, Souren LE, Torossian CL, et al.. 1999. Equilibrium and limb coordination in mild cognitive impairment and mild Alzheimer's disease. J Am Geriatr Soc, 47:463-9.

Garcia A, Thompson K, Zanibbi K, et al. 2007. Cholinesterase inhibitors and Alzheimer's disease outcomes. J Gerontol A Biol Sci Med Sci, 62:570.

Goldman WP, Baty JD, Buckles VD, et al. 1999. Motor dysfunction in mildly demented AD individuals without extrapyramidal signs. $\mathrm{Neu}$ rology, 53:956-62.

Hausdorff JM. 2004. Stride variability: beyond length and frequency. Gait Posture, 20:304.

Hausdorff JM, Yogev G, Springer S, et al. 2005. Walking is more like catching than tapping: gait in the elderly as a complex cognitive task. Exp Brain Res, 164:541-8.

Marquis S, Moore MM, Howieson DB, et al. 2002. Independent predictors of cognitive decline in healthy elderly persons. Arch Neurol, 59:601-6.

Merory JR, Wittwer JE, Rowe CC, et al. 2007. Quantitative gait analysis in patients with dementia with Lewy bodies and Alzheimer's disease. Gait Posture, 26:414-19

Morgan D, Funk M, Crossley M, et al. 2007. The potential of gait analysis to contribute to differential diagnosis of early stage dementia: current research and future directions. Can J Aging, 26:19-32.

Nutt JG, Marsden CD, Thompson PD. 1993. Human walking and higher-level gait disorders, particularly in the elderly. Neurology, 43:268-79

Nakamura T, Meguro K, Sasaki H. 1996. Relationship between falls and stride length variability in senile dementia of the Alzheimer type. Gerontology, 42:108-13.

Nakamura T, Meguro K, Yamazaki H, et al. 1997. Postural and gait disturbance correlated with decreased frontal cerebral blood flow in Alzheimer disease. Alzheimer Dis Assoc Disord, 11:132-9.

Nevitt MC, Cummings SR, Hudes ES. 1991. Risk factors for injurious falls: a prospective study. J Gerontol, 46:M164-70.

Oliver D, Hopper A, Seed P. 2003. Do hospital fall prevention programs work? A systematic review. J Am Geriatr Soc, 48:1679-89.

Oliver D, Daly F, Martin FC, et al. 2004. Risk factors and risk assessment tools for falls in hospital in-patients: a systematic review. Age Ageing, 33:122-30.
O'Keeffe ST, Kazeem H, Philpott RM, et al. 1996. Gait disturbance in Alzheimer's disease: a clinical study. Age Ageing, 25:313-16.

Pahapill PA, Lozano AM. 2000. The pedunculopontine nucleus and Parkinson's disease. Brain, 123:1767-83.

Pashler H. 1994. Dual-task interference in simple task: Data and theory. Psychol Bull, 116: 220-44.

Rimmer E, Wojciechowska M, Stave C, et al. 2005. Implications of the Facing Dementia Survey for the general population, patients and caregivers across Europe. Int J Clin Pract Suppl, 146:17-24.

Sattin RW. 1992. Falls among older persons: a public health perspective. Annu Rev Public Health, 13:489-508.

Scarmeas N, Albert M, Brandt J, et al. 2005. Motor signs predict poor outcomes in Alzheimer disease. Neurology, 64:1696-703.

Scherder E, Eggermont L, Swaab D, et al. 2007. Gait in ageing and associated dementias; its relationship with cognition. Neurosci Biobehav Rev, 31:485-97.

Shaw FE. 2002. Falls in cognitive impairment and dementia. Clin Geriatr Med, 18:159-73.

Sheridan PL, Solomont J, Kowall N, et al. 2003. Influence of executive function on locomotor function: divided attention increases gait variability in Alzheimer's disease. J Am Geriatr Soc, 5:1633-7.

Small BJ, Gagnon E, Robinson B. 2007. Early identification of cognitive deficits: preclinical Alzheimer's disease and mild cognitive impairment. Geriatrics, 62:19-23.

Squire LR, Zola-Morgan S, Cave CB, et al. 1990. Memory: organization of brain systems and cognition. Cold Spring Harb Symp Quant Biol, 55:1007-23

Tinetti ME, Speechley M, Ginter SF. 1988. Risk factors for falls among elderly persons living in the community. $N$ Engl $J$ Med, 319:1701-7.

Tinetti ME. 2003. Clinical practice. Preventing falls in elderly persons. $N$ Engl J Med, 348:42-9.

Treisman AM.1969. Strategies and models of selective attention. Psychol Rev, 76:282-99.

Van Iersel MB, Benraad CE, Rikkert MG. 2007. Validity and reliability of quantitative gait analysis in geriatric patients with and without dementia. $J$ Am Geriatr Soc, 55:632-4.

Van Iersel MB, Hoefsloot W, Munneke M, et al. 2004. Systematic review of quantitative clinical gait analysis in patients with dementia. Z Gerontol Geriatr, 37:27-32.

Verghese J, Lipton RB, Hall CB, et al. 2002. Abnormality of gait as a predictor of non-Alzheimer's dementia. $N$ Engl J Med, 347:1761-8.

Verghese J, Derby C, Katz MJ, et al. 2007a. High risk neurological gait syndrome and vascular dementia. J Neural Transm, 114:1249-52.

Verghese J, Wang C, Holtzer R, et al. 2007b. Quantitative gait dysfunction and risk of cognitive decline and dementia. J Neurol Neurosurg Psychiatry, 78:929-35.

Visser H. 1983. Gait and balance in senile dementia of Alzheimer's type. Age Ageing, 12:296-301.

Visser PJ, Verhey FR. 2007. Mild cognitive impairment as predictor for Alzheimer's disease in clinical practice: effect of age and diagnostic criteria. Psychol Med, 24:1-10.

Waldemar G, Dubois B, Emre M, et al. 2007; EFNS. Recommendations for the diagnosis and management of Alzheimer's disease and other disorders associated with dementia: EFNS guideline. Eur J Neurol, 14:e1-26.

Waite LM, Grayson DA, Piguet O, et al. 2005. Gait slowing as a predictor of incident dementia: 6-year longitudinal data from the Sydney Older Persons Study. J Neurol Sci, 229-230:89-93.

Webster KE, Merory JR, Wittwer JE. 2006. Gait variability in community dwelling adults with Alzheimer disease. Alzheimer Dis Assoc Disord, 20:37-40.

Woollacott M, Shumway-Cook A. 2002. Attention and the control of posture and gait: A review of an emerging area of research. Gait Posture, $16: 1-14$. 